\title{
THE INFINITESIMAL STABILITY OF SEMIGROUPS OF EXPANDING MAPS
}

\author{
BY \\ RICHARD SACKSTEDER
}

ABSTRACT. The concept of $C^{\infty}$ infinitesimal stability for representations of a semigroup by $C^{\infty}$ maps is defined. In the case of expanding linear maps of the torus $T^{d}$ it is shown that certain algebraic conditions assure such stability.

1. Introduction. Let $S$ be an abstract semigroup and let $\phi: S \rightarrow C^{\infty}(X, X)$ be a representation of $S$ by $C^{\infty}$ maps of a manifold $X$ so that $\phi(s t)=\phi(s) \circ \phi(t)$. Let $\pi: T(X) \rightarrow X$ be the tangent bundle of $X$. A map $\Psi: S \rightarrow C^{\infty}(X, T(X))$ is called a $C^{\infty}$ infinitesimal perturbation of $\phi$ if:

(a) $\pi \circ \Psi(s)=\phi(s)$, that is $\Psi(s)$ is a section of the pullback of $T(X)$ by $\phi(s)$, and

(b) for every $s$ and $t$ in $S$,

$$
\Psi(s t)=\Psi(s) \circ \phi(t)+D \phi(s) \circ \Psi(t) .
$$

All of the terms are sections of the pullback of $T(X)$ by $\phi(s t)$, so the addition makes sense. The motivation for the relation $(1.1)_{s t}$ is as follows: If $\phi_{\epsilon}(s)$ is a family of representations depending smoothly on the parameter $\epsilon, \phi_{0}(s)=$ $\phi(s)$ and $d \phi(s) /\left.d \epsilon\right|_{\epsilon=0}=\Psi(s)$, then (1.1)st is the result of differentiating $\phi_{\epsilon}(s t)$ $=\phi_{\epsilon}(s) \phi_{\epsilon}(t)$ at $\epsilon=0$.

An infinitesimal deformation $\Psi$ of $\phi$ is called a $C^{\infty}$ infinitesimal conjugate if there is a vector field $f$ such that for every $s$ in $S$,

$$
f \circ \phi(s)-D \phi(s) \circ f=\Psi(s) .
$$

Here every term is a section of the pullback of $T(X)$ by $\phi(s)$. The motivation for $(1.2)_{s}$ is analogous to that for $(1.1)_{s t}$. The representation $\phi$ is said to be infinitesimally $C^{\infty}$ stable if every $C^{\infty}$ infinitesimal perturbation of $\phi$ is a $C^{\infty}$ infinitesimal conjugate of $\phi$.

If $S$ is a free semigroup $\Psi(s)$ can be specified arbitrarily, except for the condition (a) above, on a free set of generators, and then (1.1) $)_{s t}$ determines $\Psi$ on

Received by the editors December 10,1974 and, in revised form, June 6, 1975 .

AMS (MOS) subject classifications (1970). Primary 58F10, 58F15; Secondary 20M20.

Key words and phrases. Stability, dynamical systems, semigroups of transformations, expanding maps. 
all of $S$. When there are relations in the semigroup, (1.1) st yields constraints on $\Psi(s)$ even if $s$ is a generator. Obviously, the more relations in $S$, the easier it is for a representation to be $C^{\infty}$ stable.

As the motivation described above suggests, the concept of infinitesimal stability is of interest because of its relevance to smooth one parameter families of deformation of representations. However, the concept is of further potential interest because it may be of use in proving the stability of representations via the technique of the Moser implicit functions theorem (cf. [3] or [6]). There are difficulties in applying the technique to stability problems for semigroups, stemming from the fact that the smoothing operators tend to destroy relations in the semigroup. I have not yet been able to overcome these difficulties for the representations considered below. A special case of the problems treated here is discussed in [4]. An alternate approach to the problem of relating infinitesimal stability to stability might be by means of an analogue of the Mather theorem (cf. [1] or [2]) applicable to representations of semigroups.

2. Special representations. It will always be assumed below that the semigroup $S$ is finitely generated by elements $s_{0}, \ldots, s_{k}$, where $s_{0}$ is central, that is $s_{0} t=t s_{0}$ for every $t$ in $S$. Of course, $s_{0} t=t s_{0}$ for all $t$ follows from the special cases $t=s_{i}, i=1, \ldots, k$. Thus, $\Psi\left(s_{0} s_{i}\right)=\Psi\left(s_{i} s_{0}\right)$ and the corresponding relations $(1.1)_{s t}$ provide the constraints on the infinitesimal deformations of representation $\phi$ of $S$.

Another specialization employed below is that $X$ will be the $d$-torus, $T^{d}=$ $R^{d} / Z^{d}$, and the representation $\phi$ will be by linear maps, that is by maps whose lifts to $R^{d}$ are linear. The following notation will be used:

$$
\begin{aligned}
N_{i} & =\text { lift of } \phi\left(s_{i}\right) \text { to } R^{d}, \\
G_{i} & =\text { lift of } \Psi\left(s_{i}\right) \text { to } R^{d} .
\end{aligned}
$$

Then $N_{i}$ is a nonsingular $d \times d$ matrix with integer entries, and $G_{i}(x) \equiv G_{i}(x+n)$ for all $x$ in $R^{d}$ and $n$ in $Z^{d}$.

Now the constraints imposed on $G_{i}$ are

$$
G_{0} \circ N_{i}-N_{i} \circ G_{0}=G_{i} \circ N_{0}-N_{0} \circ G_{i} \text {, }
$$

and $G_{i}$ is an infinitesimal conjugate if there is a solution $F$ of

$$
F \circ N_{i}-N_{i} \circ F=G_{i}
$$

where $F$ is the lift of the $f$ in (1.2)s.

Our main theorem is as follows:

THEOREM 2.1. Let $S$ be a semigroup generated by $s_{0}, \ldots, s_{k}$, where $s_{0}$ is central, and let $\phi$ be a representation of s by linear maps of $T^{d}$. Then $\phi$ is $C^{\infty}$ infinitesimally stable provided 


$$
N_{i} \text { is an expanding map }(i=0, \ldots, k) \text {, and for any } n \text { in } Z^{d} \text {, }
$$

$$
N_{i}^{*} n \equiv 0 \bmod N_{0}^{*}(i=1, \ldots, k) \text { implies } n \equiv 0 \bmod N_{0}^{*} .
$$

The condition (2.4) means that for some $\lambda>1,\left|N_{i} x\right| \geqslant \lambda|x|$ holds for every $x$ in $R^{d}$ (cf. [5]), and in (2.5) $m \equiv 0 \bmod N_{0}^{*}$ means that $m=N_{0}^{*} z$ for some $z$ in $Z^{d}$. Here the asterisk denotes transpose with respect to the usual inner product on $R^{d}$.

Actually, a somewhat stronger result than Theorem 2.1 will be proved. This result (Theorem 4.1) below states roughly that if the relations $(2.1)_{i}$ are satisfied approximately, $(2.2)_{i}$ will be satisfied approximately. This stronger version of Theorem 2.1 is proved here because it seems likely that applications to stability questions of the sort discussed at the end of the preceding section will require it.

3. Lemmas. Let $Y$ be a real finite dimensional vector space with inner product denoted by $u \cdot v$ and norm $|u|^{2}=u \cdot u$. The norm $\|F\|_{\alpha}(\alpha \geqslant 0)$ for functions $F: T^{d} \rightarrow Y$ is defined by

$$
\|F\|_{\alpha}=\sum(1+|n|)^{\alpha}\left|a_{n}\right| \text {, }
$$

where the summation is over all $n$ in $Z^{d}, F \sim \Sigma a_{n} e(n x)$ is the Fourier series for $F$, and $e(n x)=\exp (2 \pi i n \cdot x)$. Note that each $a_{n}$ is an element of $Y$. The space of $F$ 's such that $\|F\|_{\alpha}<\infty$ is a Banach space which we denote by $B_{\alpha}$.

If $L^{2}=L^{2}\left(T^{d}, Y\right)$ is the space of square integrable functions on $T^{d}$ with values in $Y$, and $t_{i}$ is a nonsingular linear map of $T^{d}$ which lifts to $N_{i}$ on $R^{d}$, $V_{t} F(x)=F\left(t_{i}(x)\right)$ defines an isometry of $L^{2}$ and bounded linear map of the function spaces $B_{\alpha}(\alpha \geqslant 0)$. If $V_{i}^{*}$ is the adjoint of $V_{i}$ on $L^{2}, V_{i}^{*}$ is a left inverse of $V_{i}$. Since $B_{\alpha} \subseteq B_{0} \subseteq L^{2}$ for $\alpha \geqslant 0, V_{i}^{*}$ acts on $B_{\alpha}$ and is easily seen to be a contraction on any $B_{\alpha}$. It is easily checked that $V_{i}^{*}$ can be written explicitly as follows:

$$
V_{i}^{*} F(x)=\left|\operatorname{det} N_{i}\right|^{-1} \sum_{z} F\left(N_{i}^{-1}(x+z)\right),
$$

where the summation is over elements $z$ of $Z^{d}$ such that $N_{t}^{-1} z$ is in some fixed fundamental domain for $T^{d}$. (Here and below we make use of the fact that functions $F$ on $T^{d}$ can be viewed as functions on $R^{d}$ satisfying $F(x)=F(x+z)$ for all $z$ in $Z^{d}$.)

If $M^{p}$ is a $p$-linear map from $R^{d}$ to $R^{d}$ and $N$ is a linear map from $R^{d}$ to $R^{d}, M^{p} N_{p}$ will denote the $p$-linear map defined by:

$$
M^{p} N_{p}\left(\xi^{1}, \ldots, \xi^{p}\right)=M^{p}\left(N \xi^{1}, \ldots, N \xi^{p}\right),
$$


where each $\xi^{j}$ is in $R^{d}$. The norm for such a map $M^{p}$ is defined in terms of a fixed norm on $R^{d}$ by:

$$
\left|M^{p}\right|=\operatorname{Sup}\left\{\left|M^{p}\left(\xi^{1}, \ldots, \xi^{p}\right)\right|: \prod_{j=1}^{p} \xi^{j} \mid \leqslant 1\right\} .
$$

LemMa 3.1. Let $N$ be an expanding $d \times d$ matrix. Then for any $\theta>0$, there is a $P=P(\theta, N)$ such that if $p \geqslant P$.

$$
\left|N \circ M^{p} N_{p}^{-1}\right| \leqslant \theta\left|M^{p}\right|
$$

holds for every p-linear map $M^{p}$.

The proof is an immediate consequence of the definition of the norm of a $p$-linear map and the fact for any expanding linear map $N,\left|N^{-1}\right|<1$.

If $G_{i}$ is as above, $G_{i}^{p}$ will denote the $p$ th derivative of $G_{i}(0 \leqslant p<\infty)$. If $p \geqslant 1, G_{i}^{p}(x)$ is at each point $x$ in $R^{d}$ a $p$-linear map from $R^{d}$ to $R^{d}$. Moreover, $G_{i}^{p}(x)=G_{i}^{p}(x+z)$ for $z$ in $Z^{d}$, so $G_{i}^{p}(x)$ can be regarded as defined for $x$ in $T^{d}$. Since $N_{j}$ is linear, the $p$ th derivative of $G_{i} \circ N_{j}$ at $x$ in $T^{d}$ is given by $V_{j} G_{i}^{p}(x) N_{j p}$. Defining $F^{p}$ analogously to $G_{i}^{p}$ and differentiating $(2.2)_{i} p$ times, gives

$$
V_{i} F^{p} N_{i p}-N_{i} \circ F^{p}=G_{i}^{p} .
$$

(The case $p=0$ is understood as (2.2) itself.)

Applying $V_{i}^{*}$ to this equation and composing each component of the multilinear part with $N_{i}^{-1}$ on the right gives

$$
F^{p}-N_{i} \circ V_{i}^{*} F^{p} N_{i p}^{-1}=V_{i}^{*} G_{i}^{p} N_{i p}^{-1}
$$

The next lemma shows the existence of solutions of equations of the same form as (3.2) $)_{i}^{p}$.

LEMмA 3.2. Let $t$ be an expanding map of $T^{d}$, suppose that the linear map $N$ is the lift of $t$ to $R^{d}$ and let $V$ be the corresponding isometry of $L^{2}\left(T^{d}\right)$. Let $B$ be a $C^{\infty}$ map from $T^{d}$ to $R^{d}$ and let $B^{q}$ denote its qth derivative. Then there is a unique solution $F$ of

$$
F-N \circ V^{*} F=V^{*} B,
$$

whose qth derivative $F^{q}$ satisfies

$$
F^{q}-N \circ V^{*} F^{q} N_{q}^{-1}=V^{*} B^{q} N_{q}^{-1} .
$$

For sufficiently large $\alpha,\|F\|_{\alpha} \leqslant$ const $\|B\|_{\alpha}$.

(Here $V^{*}$ on the right side of $(3.3)^{q}$ and $(3.3)^{0}$ has no effect since $V^{*}$ is onto. The relations are written as they are to emphasize their similarity to (3.2) $)_{i}^{p}$.) 
Proof. Define

$$
F^{p}=\sum_{s=1}^{\infty} N^{s-1} \circ V^{-s} B^{p} N_{p}^{-s},
$$

where $V^{-s}$ means $\left(V^{*}\right)^{s}$. If $p$ is large enough the series converges uniformly on $T^{d}$ by Lemma 3.1. For such large $p$, the formal computation showing that $F^{p}$ satisfies $(3.3)^{p}$ is justified.

The difference $\Delta^{p}$ of two solutions of $(3.3)^{p}$ would have to satisfy $\Delta^{p}=$ $N \circ V^{-1} \Delta^{p} N_{p}^{-1}$ hence by iteration $\Delta^{p}=N^{s} \circ V^{-s} \cdot \Delta^{p} N_{p}^{-s}$. Applying Lemma 3.1 shows that for large $p$ the right side goes uniformly to 0 as $s \rightarrow \infty$, hence the solution $F^{p}$ of $(3.3)^{p}$ is unique for large $p$ at least.

Formally it is clear from the explicit formula for $V^{*}$ given above that $F^{q+1}$ is the derivative of $F^{q}$ and there is no difficulty in justifying the formalities for $q \geqslant p$.

The proposition in the appendix shows how $F$ and $F^{q}$ for $0 \leqslant q<p$ are constructed. Note that here $F(0)$ cannot be chosen arbitrarily. To see this let $F_{0}(x)$ be the antiderivative of $F^{1}(x)$ satisfying $F_{0}(0)=0$. Setting $F(x)=F(0)$ $+F_{0}(x)$ and substituting in (3.3) ${ }^{0}$ with $x=0$ gives

$$
(I-N) \circ F(0)=N \circ\left(V^{*} F_{0}\right)(0)+\left(V^{*} B\right)(0) .
$$

Since $I-N$ is invertible, $F(0)$ is uniquely determined by this relation.

The estimates for $\|F\|_{\alpha}$ for $\alpha \geqslant p$ follow from those in the proposition in the appendix and simple estimates for $F(0)$.

The next lemma depends on the relation

$$
G_{0} \circ N_{i}-N_{i} \circ G_{0}-G_{i} \circ N_{0}+N_{0} \circ G_{i}=R_{i} \text {, }
$$

where $R_{i}$ is a map from $R^{d}$ to $R^{d}$ satisfying $R_{i}(x) \equiv R_{i}(x+z)$ for $x$ in $R^{d}$ and $z$ in $Z^{d}$. The relation $(3.5)_{i}^{0}$ generalizes $(2.1)_{i}$. Differentiating it $p$ times gives

$$
V_{i} G_{0}^{p} N_{i p}-N_{i} \circ G_{0}^{p}-V_{0} G_{i}^{p} N_{0 p}+N_{0} \circ G_{i}^{p}=R_{i}^{p} \text {. }
$$

LEMMA 3.3. Assume (3.2) $)_{0}^{p}$ and (3.5) ${ }_{i}^{p}$ for $p \geqslant 0$ and some $i=1, \ldots, k$. Let $H_{i}^{p}=F^{p} N_{i p}-N_{i} \circ V_{i}^{*} F^{p}-V_{i}^{*} G_{i}^{p}$. Then

$$
H_{i}^{p} N_{0 p}-N_{0} \circ V_{0}^{*} H_{i}^{p}=-V_{0}^{*} V_{i}^{*} R_{i}^{p},
$$

hence, by Lemma 3.2, $\left\|H_{i}\right\|_{\alpha} \leqslant$ const $\left\|R_{i}\right\|_{\alpha}$. In particular, $F^{p}$ satisfies (3.2) I $_{i}$ if $R_{i}=0$.

PROOF. Let $h_{0}=V_{0} F^{p} N_{0 p}-N_{0} \circ F^{p}$ and $h_{i}=V_{i} F^{p} N_{i p}-N_{i} \circ F^{p}$. It is easy to check that

$$
V_{0} h_{i} N_{0 p}-N_{0} \circ h_{i}-V_{i} h_{0} N_{i p}+N_{i} \circ h_{0}=0 .
$$


Letting $Q_{i}=h_{i}-G_{i}^{p}$ and $Q_{0}=h_{0}-G_{0}^{p},(3.5)_{i}^{p}$ and (3.7) give

$$
V_{0} Q_{t} N_{0 p}-N_{0} \circ Q_{i}-V_{i} Q_{0} N_{i p}+N_{i} \circ Q_{0}=-R_{i}^{p} \text {. }
$$

Applying $V_{0}^{*} V_{i}^{*}$ to both sides of (3.8) gives

$$
V_{i}^{*} Q_{i} N_{0 p}-N_{0} \circ V_{0}^{*} V_{i}^{*} Q_{i}=-V_{0}^{*} V_{i}^{*} R_{i}^{p},
$$

since (3.2) $)_{0}^{p}$ implies that $V_{0}^{*} Q_{0}=0$. But since $H_{i}^{p}=V_{i}^{*} Q_{i},(3.9)$ is the same as (3.6).

4. Proof of Theorem 2.1. The special case in which $R_{i}=0$ in $(3.5)_{i}^{0}$ in the following theorem implies Theorem 2.1.

Theorem 4.1. Assume (2.4), (2.5), and (3.5) ${ }_{i}^{0}$ for $i=1, \ldots, k$. Then the solution $F$ of (3.2) $)_{0}^{0}$ satisfies

$$
F \circ N_{i}-N_{i} \circ F=G_{i}+\epsilon_{i} \quad(i=0, \ldots, k),
$$

where for large $\alpha_{1}\left\|\epsilon_{i}\right\|_{\alpha} \leqslant$ const $\left(\Sigma_{j=1}^{k}\left\|R_{j}\right\|_{\alpha}\right)$, for $i=0, \ldots, k$.

Proof. Let the Fourier series of $Q_{0}, Q_{i}$, and $R_{i}^{p}$ be

$$
Q_{0} \sim \sum a(n) e(n x), \quad Q_{i} \sim \sum b_{i}(n) e(n x), \quad R_{i}^{p} \sim \sum c_{i}(n) e(n x),
$$

where $Q_{0}$ and $Q_{i}$ are as in the last section. Then

$$
H_{i}=V_{i}^{*} Q_{i} \sim \sum b_{i}\left(n / N_{i}^{*}\right) e(n x),
$$

where conventionally $b_{i}\left(n / N_{i}^{*}\right)=0$ if $n \neq 0 \bmod N_{i}^{*}$ and $b_{i}\left(n / N_{i}^{*}\right)=b_{i}(m)$ if $n=N_{i}^{*} m$ for $m$ in $Z^{d}$. Defining $b_{i}\left(n / N_{0}^{*}\right)$ and $a\left(n / N_{i}^{*}\right)$ analogously, one sees that (3.8) gives

$$
-c(n)=N_{i} \circ a(n)-a\left(n / N_{i}^{*}\right) N_{i p}+b_{i}\left(n / N_{0}^{*}\right) N_{0 p}-N_{0} \circ b_{i}(n) .
$$

Note that $V_{0}^{*} Q_{0}=0$ implies that

$$
a(n)=0 \quad \text { if } n \equiv 0 \bmod N_{0}^{*} \text {. }
$$

Now suppose that $m$ is such that $a(m) \neq 0$, hence $m \neq 0 \bmod N_{0}^{*}$ by (4.3). The assumption (2.5) then implies that for some $i, N_{i}^{*} m \neq 0 \bmod N_{0}^{*}$ and (4.2) gives

$$
a(m)=N_{i} \circ a\left(N_{i}^{*} m\right) N_{i p}^{-1}-N_{0} \circ b_{i}\left(N_{i}^{*} m\right) N_{i p}^{-1}+c_{i}\left(N_{i}^{*} m\right) N_{i p}^{-1}
$$

since $b_{i}\left(N_{i}^{*} m / N_{0}^{*}\right)=0$.

Assume from now on that $p$ is large enough so that Lemma 3.1 holds for $N=N_{1}, \ldots, N_{k}$ and $\theta$ such that $\theta k<\beta<1$.

Then (4.4) implies that 


$$
|a(m)| \leqslant \theta\left\{\left|a\left(N_{i}^{*} m\right)\right|+K\left(\left|b_{i}\left(N_{i}^{*} m\right)\right|+\left|c_{i}\left(N_{i}^{*} m\right)\right|\right)\right\},
$$

where $K$ is a constant. If $B(m)$ is defined by

then (4.5) implies

$$
B(m)=\sum_{j=1}^{k}\left\{\left|b_{j}\left(N_{j}^{*} m\right)\right|+\left|c_{j}\left(N_{j}^{*} m\right)\right|\right\},
$$

$$
|a(m)| \leqslant \theta\left\{\left|a\left(N_{i}^{*} m\right)\right|+K B(m)\right\} .
$$

Continuing in this manner, one obtains a sequence $M_{1}, M_{2}, \ldots$ such that each $M_{j}$ is $N_{1}^{*}$ or $N_{2}^{*}, \ldots$, or $N_{k}^{*}$ and if $S_{v}=M_{v}{ }^{\circ} M_{v-1}{ }^{\circ} \ldots \circ M_{1}$,

and letting $v \rightarrow \infty$ gives

$$
|a(m)| \leqslant \theta^{v}\left|a\left(S_{v} m\right)\right|+K \sum_{j=1}^{v} \theta^{j} B\left(S_{j-1} m\right)
$$

$$
|a(m)| \leqslant K \sum_{j=1}^{\infty} \theta^{\prime} B\left(S_{j-1} m\right) .
$$

For a fixed integer $j$, any $n$ in $Z^{d}$ can appear as an $S_{j} m$ for some $m$ and $S_{j}$ in at most $k^{j}$ ways, hence (4.7) implies that

$$
\begin{aligned}
\left\|Q_{0}\right\|_{\alpha} & =\sum_{m}(1+|m|)^{\alpha}|a(m)| \leqslant K \sum_{n} \sum_{j=0}^{\infty}(1+|n|)^{\alpha}(k \theta)^{j} B(n) \\
& \leqslant K(1-\beta)^{-1} \sum_{n}(1+|n|)^{\alpha} B(n) .
\end{aligned}
$$

The definition of $B(n)$ and Lemma 3.3 imply that

hence (4.8) gives

$$
\sum_{n}(1+\ln \mid)^{\alpha} B(n) \leqslant \text { const } \sum_{i=1}^{k}\left\|R_{i}^{p}\right\|_{\alpha}
$$

$$
\left\|Q_{0}\right\|_{\alpha} \leqslant \text { const } \sum_{i=1}^{k}\left\|R_{i}^{p}\right\|_{\alpha} .
$$

Applying $V_{0}^{*}$ to (3.8) and using (4.9) shows that

$$
Q_{i}-N_{0} \circ V_{0}^{*} Q_{i}=\lambda_{i}^{p},
$$

where $\left\|\lambda_{i}^{p}\right\|_{\alpha} \leqslant$ const $\left\|R_{i}^{p}\right\|_{\alpha}$, by $V_{0}^{*} Q_{0}=0$ and the boundedness of $V_{0}^{*}$ in \|\|$_{\alpha}$. Applying Lemma 3.2 to (4.10) shows that for $\alpha \geqslant p$.

$$
\left\|Q_{i}\right\|_{\alpha} \leqslant \text { const }\left\|R_{i}^{p}\right\|_{\alpha},
$$

which together with (4.9) is the assertion of the theorem.

APPENDIX. Integration on $T^{d}$. Let $Y$ be a finite dimensional vector space. Here $Y$ will almost always be one of the spaces $L_{s}^{q}\left(R^{d} ; R^{d}\right)$, the space of symmetric $q$-linear maps from $R^{d}$ to $R^{d}$. Let the continuous map $G: T^{d} \times R^{d} \rightarrow$ $Y$ be linear in the $R^{d}$ variable. Such a map is said to be integrable (or locally integrable) if for every smooth closed curve $\gamma$ (or every smooth closed curve 
$\gamma \sim 0)$ on $T^{d}$ defined by the function $x(t)$,

$$
\int_{\gamma} G\left(x(t), x^{\prime}(t)\right) d t=0 .
$$

If $H(0) \in Y$ is specified and $G$ is integrable, there is a unique $H: T^{d} \rightarrow Y$ whose derivative is $G$.

There is a simple analogue of classical calculus term by term integration theorems for functions and their derivatives defined on $T^{d}$.

Proposition. Let $A_{1}, A_{2}, \ldots$ be a sequence of functions mapping $T^{d}$ to $R^{d}$ and let $A_{s}^{p}$ denote the pth derivative of $A_{s}$. If for some $p$ the series $\Sigma_{s=1}^{\infty}\left|A_{s}^{p}(x)\right|$ converges uniformly, there are uniquely defined functions $F^{q}: T^{d}$ $\rightarrow L_{s}^{q}\left(R^{d} ; R^{d}\right),(q=1, \ldots, p)$ such that $F^{q}$ is the derivative of $F^{q-1}$ and $F^{p}(x)=\sum_{s=1}^{\infty} A_{s}^{p}(x)$. Also, if $F(0)$ is given, there is a uniquely determined $F$ : $T^{d} \rightarrow R^{d}$ such that $F^{q}$ is the qth derivative of $F$. Moreover, for $\alpha \geqslant p$,

$$
\|F-F(0)\|_{\alpha} \leqslant \text { const }\left\|F^{p}\right\|_{\alpha-p} .
$$

The proof only needs to be sketched, since it is classical. The series for $F^{p}$ converges uniformly and absolutely. Viewing $F^{p}$ as a map from $T^{d} \times R^{d}$ to $L_{s}^{p-1}\left(R^{d} ; R^{d}\right)$ one sees that $F^{p}$ is integrable in the above sense, since each term is. Therefore, $F^{p}$ has an antiderivative which will be locally integrable by the symmetry of $A^{p}$. One and only one choice of the constant of integration makes the antiderivative into an integrable function in the above sense.

Calling the integrated series with the choice of constant that makes it integrable $F^{p-1}$, one is in a position to continue the argument in this way until $F^{1}$ has been obtained. At the final step, integrating $F^{1}$ to obtain $F$, there is no preferred choice of the constant of integration, so $F(0)$ can be specified arbitrarily. The estimates for $\|F-F(0)\|_{\alpha}$ are easy to check.

\section{REFERENCES}

1. M. Golubitsky and V. Guillemin, Stable mappings and their singularities, Graduate Texts in Math., vol. 14, Springer-Verlag, New York and Heidelberg, 1973. MR 49 \#6269.

2. J. N. Mather, Stability of $C^{\infty}$ mappings. II. Infinitesimal stability implies stability, Ann. of Math. (2) 89 (1969), 254-291. MR 41 \#4582.

3. J. Moser, $A$ rapidly convergent iteration method and non-linear differential equations. II, Ann. Scuola Norm. Sup. Pisa (3) 20 (1966), 499-535. MR 34 \$6280.

4. R. Sacksteder, Abelian semi-groups of expanding maps, Recent Advances in Topological Dynamics, Lecture Notes in Math., vol. 318, Springer-Verlag, New York and Berlin, 1973, pp. 235-248.

5. M. Shub, Endomorphisms of compact differentiable manifolds, Amer. J. Math. 91 (1969), 175-199. MR 39 \#2169.

6. S. Sternberg, Celestial mechanics. Part II, Benjamin, New York, 1969.

DEPARTMENT OF MATHEMATICS, CITY UNIVERSITY OF NEW YORK, NEW YORK, NEW YORK 10036 